\title{
3D Vector Reconstruction of the Typical Cervical Vertebra from Anatomical Sections of Korean Visible Human at the Laboratory of Clinical and Digital Anatomy of Paris Descartes University
}

\author{
Abdoulaye Kanté1,2*, Jean François Uhl' ${ }^{1}$, Mariam Daou ${ }^{2,3}$, Vincent Delmas ${ }^{1}$, J. S. Park ${ }^{4}$, \\ B. S. Chung ${ }^{4}$ Babou Ba², Nouhoum Ongoïba ${ }^{2}$ \\ ${ }^{1}$ Anatomy Laboratory, University of Paris 5, Paris, France \\ ${ }^{2}$ Anatomy Laboratory, University of Sciences, Techniques and Technologies of Bamako, Bamako, Mali \\ ${ }^{3}$ Department of Neurology, Gabriel Touré University Hospital in Bamako, Bamako, Mali \\ ${ }^{4}$ Department of Anatomy, Dongguk University School of Medicine, Dongguk, Republic of Korea \\ Email: *kanteim@yahoo.fr
}

How to cite this paper: Kanté, A., Uhl, J.F., Daou, M., Delmas, V., Park, J.S., Chung, B.S., Ba, B. and Ongoïba, N. (2020) 3D Vector Reconstruction of the Typical Cervical Vertebra from Anatomical Sections of Korean Visible Human at the Laboratory of Clinical and Digital Anatomy of Paris Descartes University. Forensic Medicine and Anatomy Research, 8, 55-63. https://doi.org/10.4236/fmar.2020.83006

Received: April 15, 2020

Accepted: June 28, 2020

Published: July 29, 2020

Copyright $\odot 2020$ by author(s) and Scientific Research Publishing Inc. This work is licensed under the Creative Commons Attribution International License (CC BY 4.0).

http://creativecommons.org/licenses/by/4.0/ (c) (i) Open Access

\begin{abstract}
Aim: To carry out a 3D vector reconstruction of the typical cervical vertebra from anatomical sections of the "Korean Visible Human" for educational purposes. Material and Methods: The anatomical subject was a 33-year-old Korean man who died of leukemia. He was $164 \mathrm{~cm}$ tall and weighed $55 \mathrm{~kg}$. This man donated his body to science. Her body was frozen and cut into several anatomical sections after an MRI and CT scan. These anatomical sections were made using a special saw called a $0.2 \mathrm{~mm}$ thick cryomacrotome. Thus 8100 cuts were obtained. Only the sections numbered 940 to 1200 were used for our study. A segmentation by manual contouring of the different parts of the typical cervical vertebra was made using the software Winsurf version 3.5 on a laptop PC running Windows 7 equipped with a Ram of 8 gigas. Results: Our $3 \mathrm{D}$ vector model of the typical cervical vertebra is easily manipulated using the Acrobat 3DPDF interface. Each part of the vertebra accessible in a menu can be displayed, hidden or made transparent, and 3D labels are available as well as educational menus for learning anatomy. Conclusion: This original work constitutes a remarkable educational tool for the anatomical study of the typical cervical vertebra and can also be used as a 3D atlas for simulation purposes for training in therapeutic gestures.
\end{abstract}




\section{Keywords}

Three-Dimensional Anatomy, Korean Human Visible, Modeling of the Typical Cervical Vertebra, Virtual Reality, 3D Reconstruction, Virtual Dissection, Surgical Simulation, Surgical Training

\section{Introduction}

Training in human anatomy is essential at all stages of medical practice: clinical examination, interpretation of medical images and surgery are based on knowledge of the anatomy of the human body. The acquisition of these skills is first theoretical then practical with dissection. Unfortunately, the provision of subjects for this stage of learning by dissection remains problematic, in general in the countries of the South and in particular in Mali, sometimes letting certain professionals start their careers with little experience in this field. Sectioned images of the human body are very useful because of their high resolutions and natural colors compared to CT scans and magnetic resonance imaging [1]. The images available include those from the Visible Human Project (VHP, male and female) conducted in the United States [1]; the Chinese human "Visible" (CVH, man and woman) [2]; the Chinese virtual human (VCH, man and woman) [3]; and the Korean "Visible" woman (VK; whole male body, male head, and female pelvis) [4].

The sectioned images of the VHP, CVH and VK males were used in several ways: for the creation of atlases [5], navigation software [6] [7] and the virtual dissection software [8] and allowed access free and free to three-dimensional models in PDF atlas files [7] [9]. In addition, cross-sectional images of VK have been used so that the radiology dose conversion coefficients are calculated virtually [10]. However, the use of prepared female sectioned images has been limited for the following reasons:

- In VHP images, degeneration of the uterus and ovaries was observed because the subject was post-menopausal (59 years), and the lateral edges of the two arms could not be used due to the subject's overweight.

- The image quality has not been optimal due to the limited performance of the digital camera and the personal computer used [11] [12].

- In addition to this, gaps in the images appeared in the digital atlases.

In $\mathrm{CVH}$ and $\mathrm{VCH}$ images, small pixel size images $(>0.1 \mathrm{~mm})$ and 24 bits color were made, but the colors of the living body could not be represented because a fixative had been injected into the body and a red dye was infused into the arteries [13]. If there were high-quality sectional images of a whole male body, they would be very useful, like images of female bodies.

In this context, we initiated this work which constitutes a part of our thesis of science relating to the $3 \mathrm{D}$ vector reconstruction of the ventral region of the neck from the anatomical sections of Korean Visible Human (KVH). 
This article deals with 3D vector reconstruction of the typical cervical vertebra.

\section{Materials and Methods}

Our study was carried out in the Research, Development, Imaging and Anatomy Unit (URDIA) EA 4465 at the Laboratory of Clinical and Digital Anatomy of the University Paris Descartes. It was conducted from 1 to 30 June 2020.

The anatomical sections of a 33-year-old Korean man who died of leukemia who donated his body were made in 2010 after an MRI and CT scan. A cryomacrotome made it possible to cut $0.2 \mathrm{~mm}$ thick sections of the frozen body, i.e. 8100 sections (Figure 1: showing photographs of KVH anatomical sections).

Only the sections numbered 940 to 1200 were used for our study (Figure 2 and Figure 3).

A segmentation by manual contouring of the different parts of the typical cervical vertebra was done using the software Winsurf version 3.5 on a laptop PC running Windows 7 equipped with an Ram of 8 gigas (Figure 4).

\section{Result}

Our methodology allowed us to build the typical cervical vertebra. We present him under the different presentations used for his teaching as it happens in the amphitheater (Figures 5-10).

\section{Discussion}

This article was made from anatomical sections of Korean Visible Human in order to achieve in the best possible way, a 3D atlas of the cervical vertebra type, dynamic and detailed.

Our work therefore consisted in recognizing the anatomical structures of the typical cervical vertebra on these sections and in a more tedious work of contouring in order to obtain the most realistic models possible. Our methodology is quite similar to that of the Korean team, which used segmentation instead of manual contouring (Figure 11: segmented sections used by the Korean team).

The advantage of this work is essentially based on the fact that the entire contouring work and therefore the entire $3 \mathrm{D}$ vector reconstruction of the typical cervical vertebra, was carried out using real sections of the human body. This results in a major increase in the precision and reliability inherent in the results presented above.

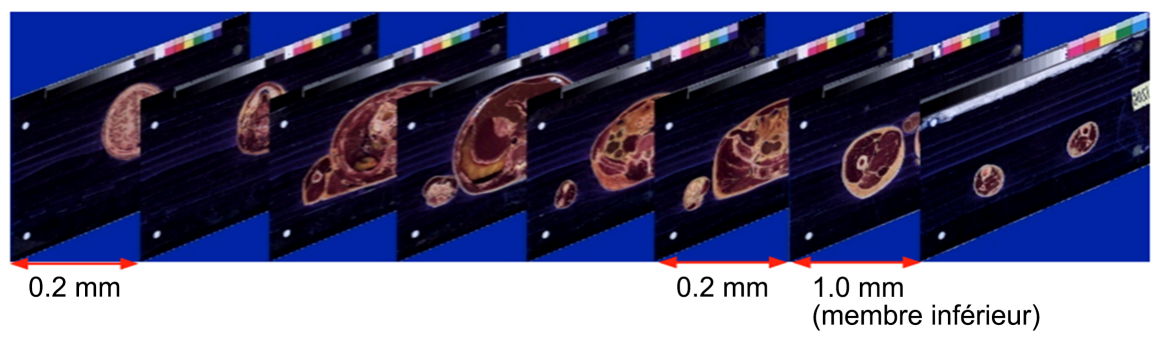

Figure 1. Showing photographs of KVH anatomical sections. 


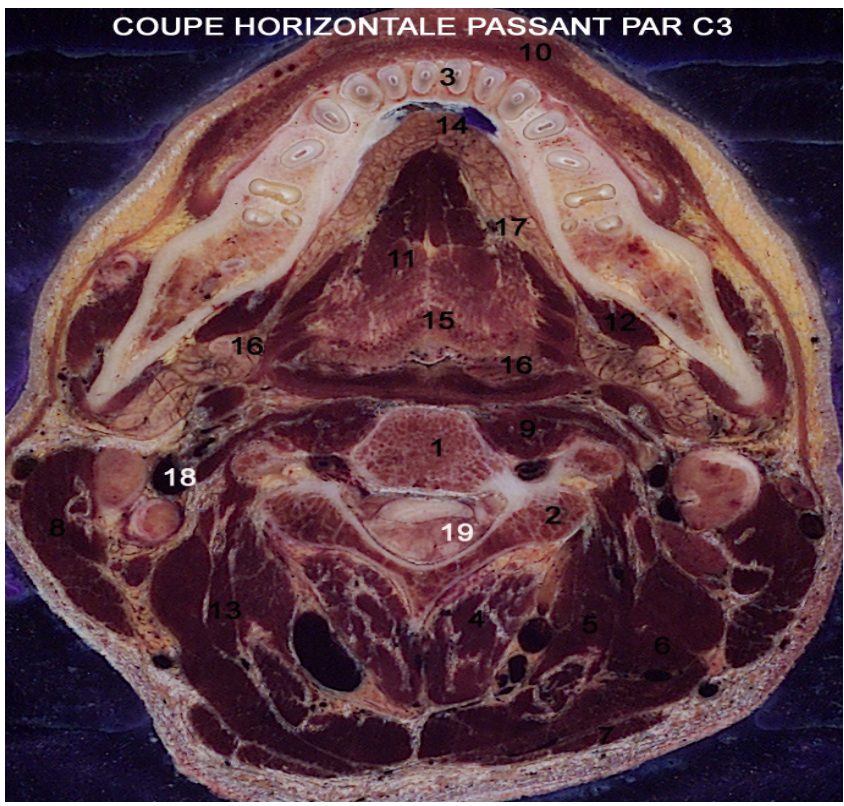

Figure 2. Anatomical section through the third cervical vertebra (C3). 1. Body of the third cervical vertebra; 2 . Joint process of $\mathrm{C} 3$; 3 . Mandible 4 . Spinous transverse neck muscle ; 5 . Semi-spiny muscle of the head; 6 . Splenius muscle of the head 7. Trapezius muscle ; 8. Sterno-cleido-mastoid muscle ; 9. Long neck muscle ; 10 . Lowering muscle of the lower lip; 11. Genio-glosseous muscle; 12. Mylohyoid muscle; 13. Elevating scapula muscle; 14. Lingual brake; 15. Epiglottis; 16. Piriform sinus; 17. Lingual artery; 18. Internal jugular vein; 19 . Cervical spinal cord.

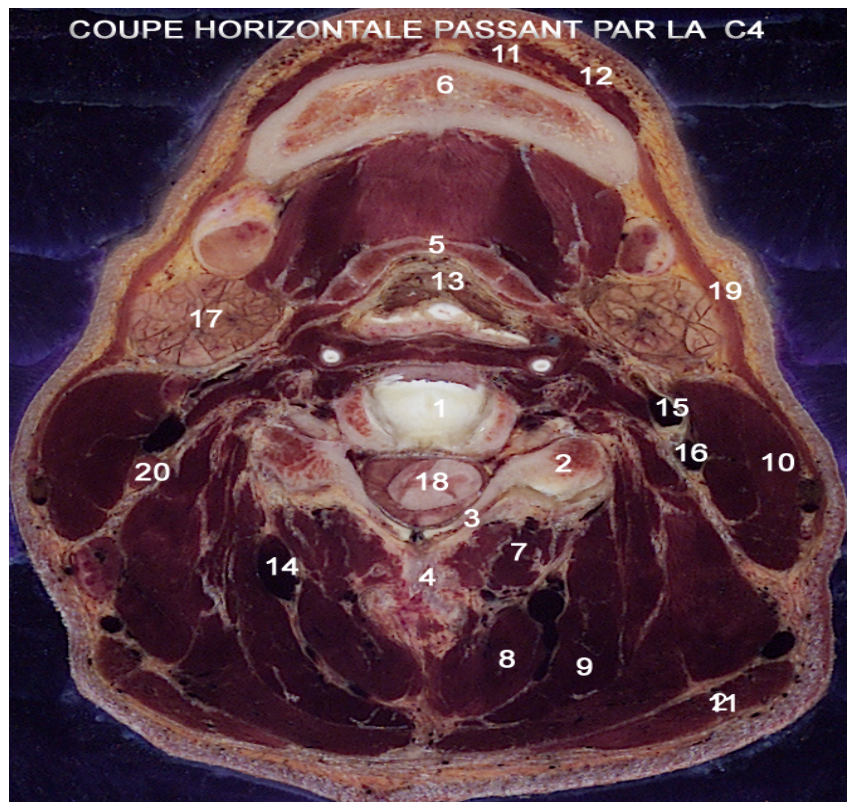

Figure 3. Anatomical section through the fourth cervical vertebra (C4). 1. Vertebral body; 2 . Transversal process; 3. Blade; 4 . Thorny process; 5 . Body of the hyoid bone 6 . Mandible; 7. Spiny transverse neck muscle; 8. Semi-spiny muscle of the head; 9. Splenius muscle of the head; 10. Sterno-cleido-mastoid muscle; 11. Genio-hyoid muscle; 12. Mylohyoid muscle; 13. Voice cord; 14. Deep cervical artery; 15. External carotid artery; 16. Internal carotid artery; 17. Sub mandibular gland; 18. Cervical cord; 19. Skin muscle; 20. Jugular lymph node; 21 . Trapezius muscle. 

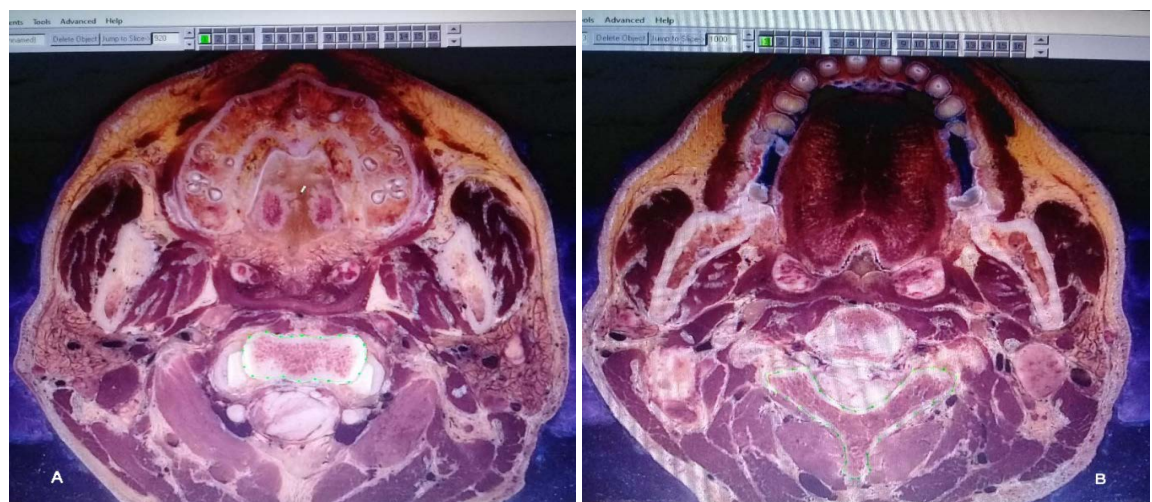

Figure 4. Interface screen of the Winsurf software (version 3.5) to draw the limits of the vertebral body and the posterior arch of the typical cervical vertebra (green line and points) on anatomical section number 940 . This is done with the pen tool using the green channel.

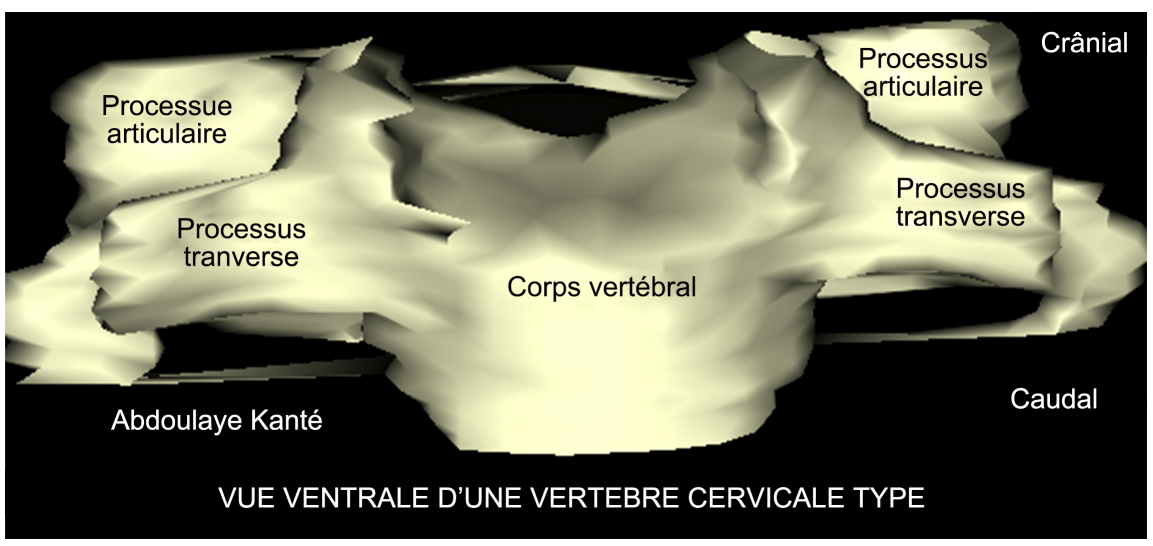

Figure 5. 3D vector reconstruction of the cervical vertebra with Winsurf software: ventral view. Joint process; Transversal process; Vertebral body.

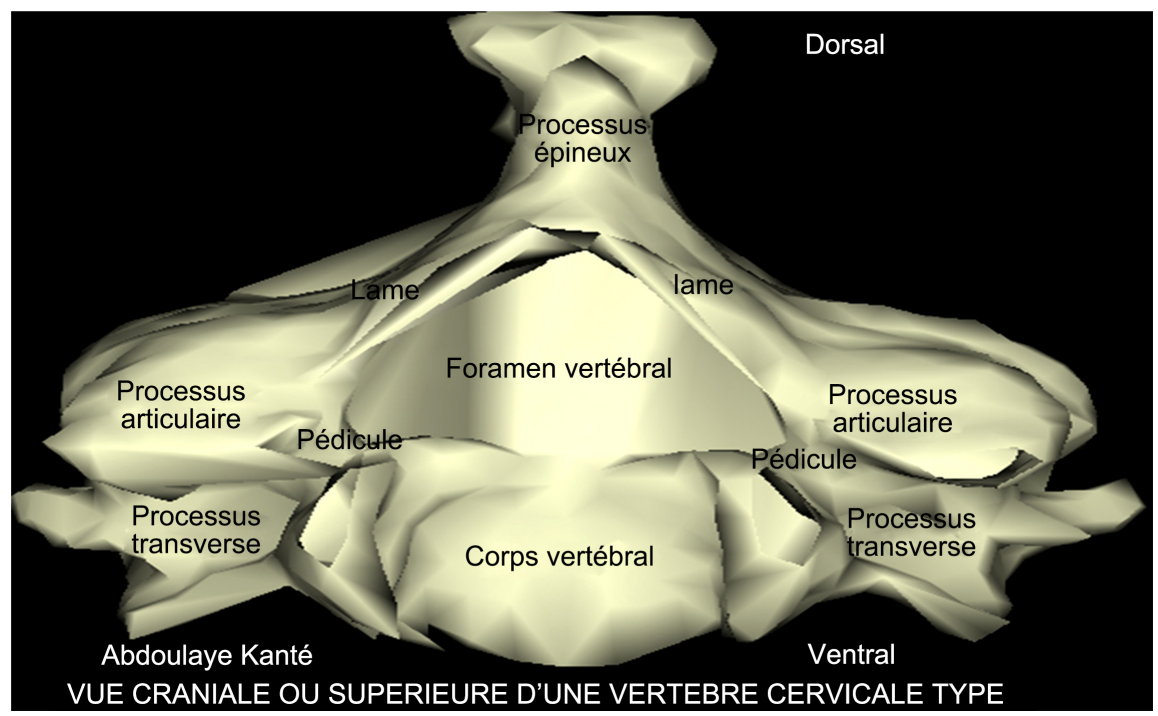

Figure 6. 3D vector reconstruction of the cervical vertebra with Winsurf software: cranial or superior view. Joint process; Transversal process; Vertebral body; Thorny process; Pedicle; Blade; Vertebral foramen. 


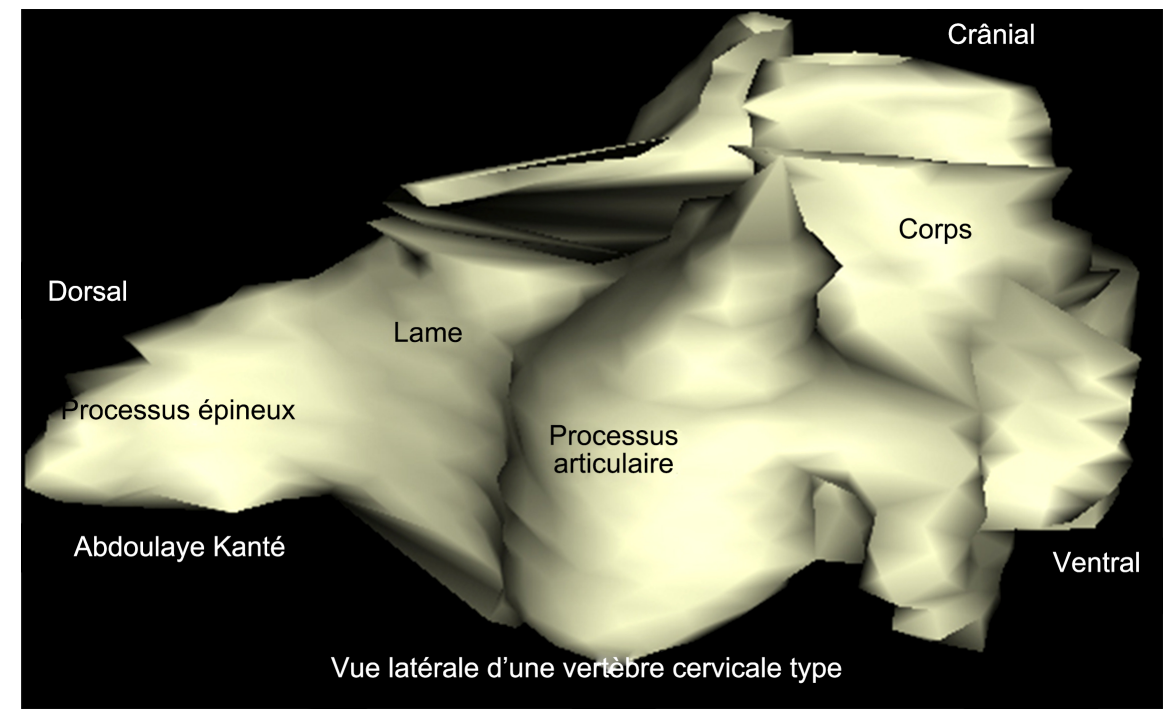

Figure 7. 3D vector reconstruction of the typical cervical vertebra with Winsurf software: side view. Joint process; Transversal process; Vertebral body; Thorny process; Pedicle; Blade; Vertebral foramen.

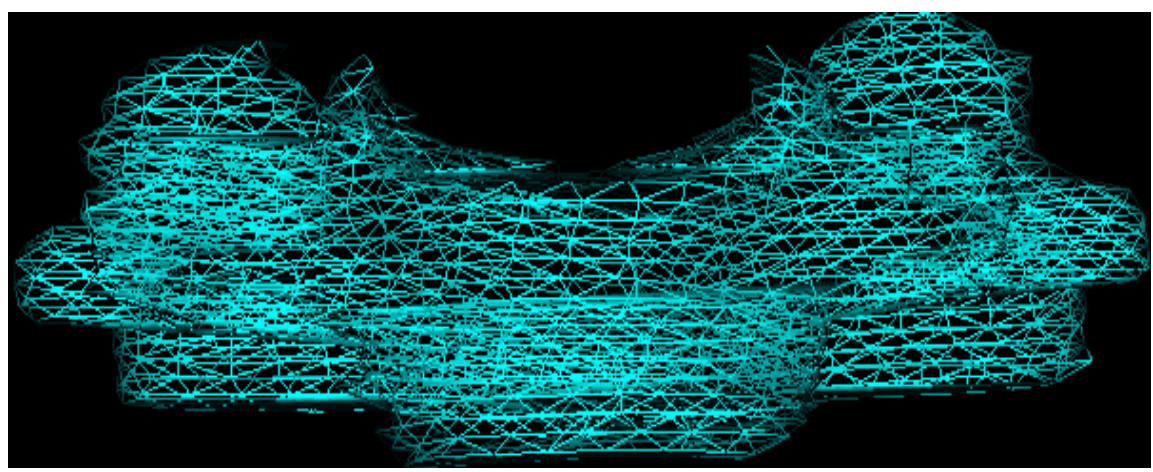

Figure 8. Wireframe representation of the typical cervical vertebra with Winsurf software.

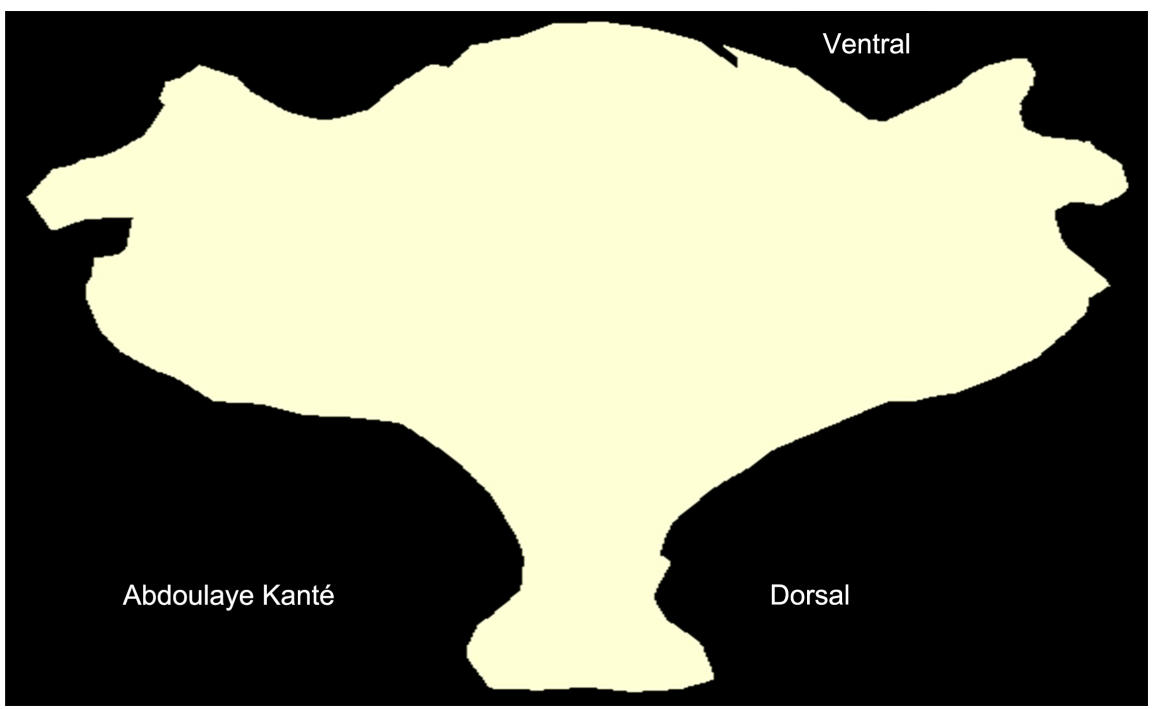

Figure 9. Gloss representation of the typical cervical vertebra. 

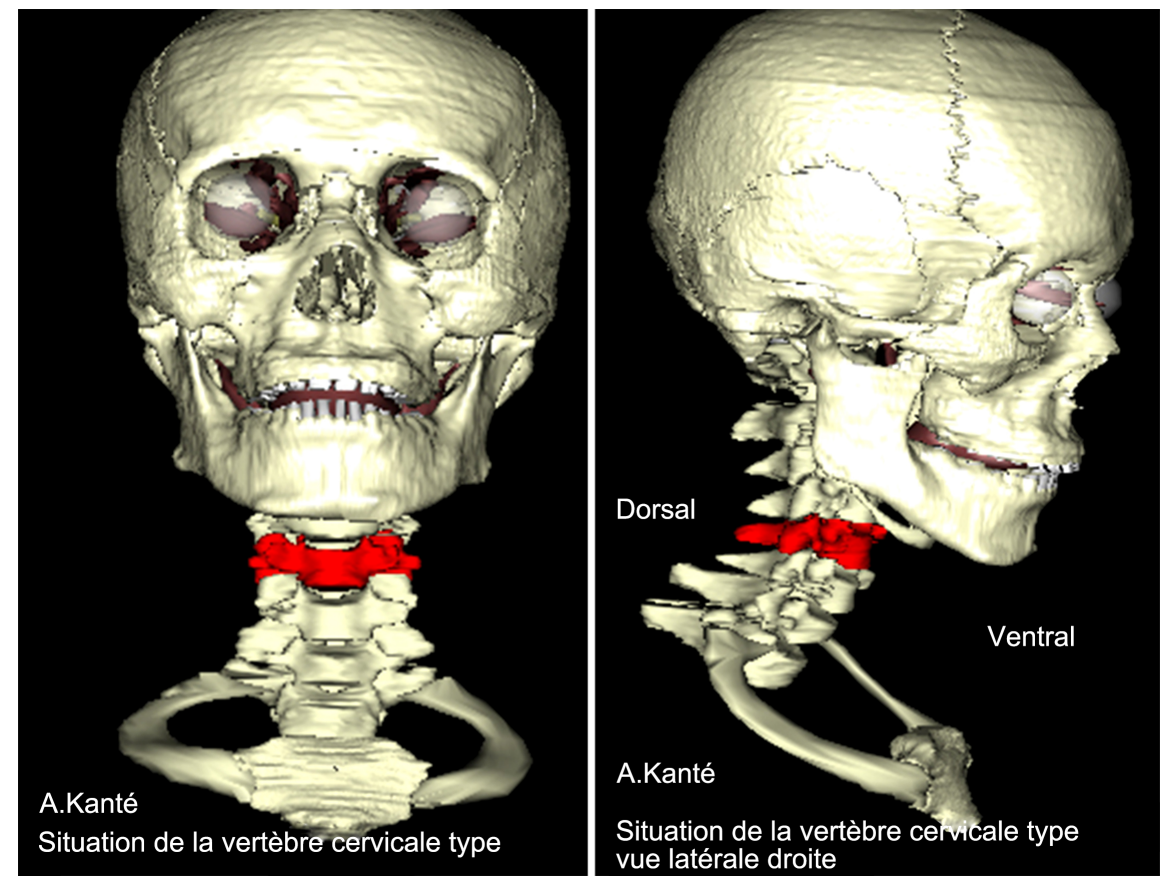

Figure 10. Situation of the typical cervical vertebra at the neck.

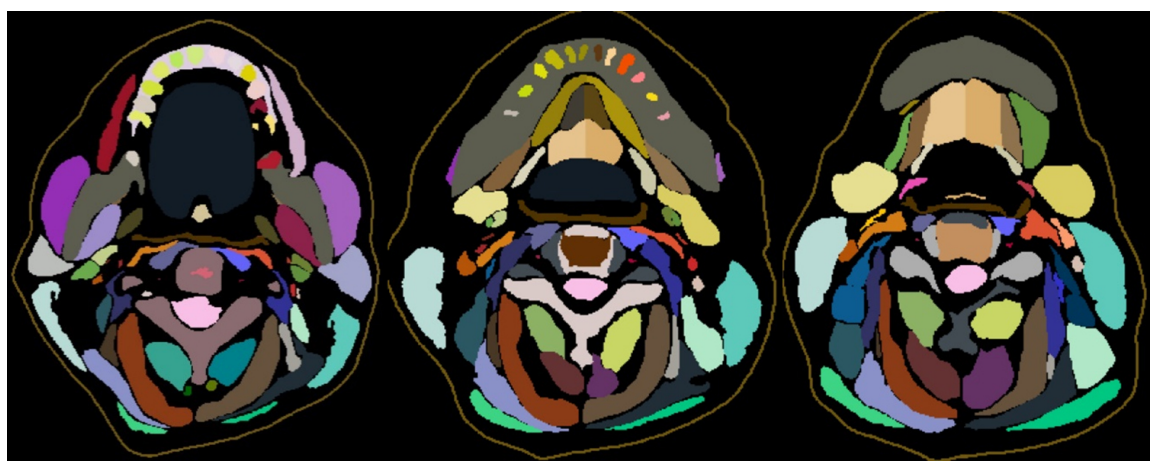

Figure 11. Segmented sections used by the Korean team.

Indeed, reconstructions of the typical cervical vertebra from digital procedures such as CT scans may prove to be somewhat disappointing in the sense that certain structures are absent and others that are difficult to distinguish. In contrast to this process, this contouring work is based on a manual, analog segmentation process under our supervision and not that of an automaton, which reduces the risk of anatomical errors in reconstruction.

The second advantage is based on the fact that better precision as well as the possibility of individualization of the different parts of the standard cervical vertebra favors a massive application in the university field thus contributing to a better understanding by medical students and other fields. In addition, it is essential to underline that this application is not restricted to the university field but can also be the support of a "Surgical Training" thus allowing a continuous training of the surgeons and a fortiori an improvement of their aptitude in their practices daily. 
Finally, it must be noted that "Winsurf" and Acrobat 3D PDF are particularly easy to use software which is not the case with other 3D modeling and manual segmentation software. In addition, they offer fairly wide ranges of textures which further increase the realism that we can bring to our final work.

Although the "Winsurf" software has made it possible to reproduce the typical cervical vertebra fairly faithfully, there are nevertheless some shortcomings.

The main disadvantage of this software is the time required to achieve the desired result. Indeed, this is a tedious contouring work of several months on several anatomical sections where sometimes only the section-by-section analysis was possible. To this are added the different objects that had to be created in order to be able to individualize the edges of the cervical vertebra, which increased the number of cuts to which it was necessary to return each time.

Unfortunately, there is no miracle cure allowing a reduction of this working time if it is not a great motivation and an unprecedented personal investment.

\section{Conclusion}

Our 3D vector modeling of the typical cervical vertebra constitutes a remarkable educational tool for teaching the anatomy of the typical cervical vertebra and can also be used as a 3D atlas for simulation purposes for training in therapeutic gestures.

\section{Conflicts of Interest}

The authors do not declare any conflict of interest concerning the publication of this document.

\section{References}

[1] Ackerman, M.J. (1991) The Visible Human Project. A Resource for Education. Academic Medicine, 74, 667-670. https://doi.org/10.1097/00001888-199906000-00012

[2] Cho, Z.H. (2009) 7.0 Tesla MRI Brain Atlas, in Vivo Atlas with Cryomacrotome Correlation. Springer, New York. https://doi.org/10.1007/978-1-60761-154-7

[3] Cho, Z.H., Calamate, F. and Chi, J.G. (2012) 7.0 Tesla MRI Brain White Matter Atlas. Panmun, Seoul., 95, 2670-2673.

[4] Chung, B.S., Shin, D.S., Brown, P., Choi, J. and Chung, M.S. (2015) Virtual Dissection Table Including the Visible Korean Images, Complemented by Free Software of the Same Data. International Journal of Morphology, 33, 440-445. https://doi.org/10.4067/S0717-95022015000200006

[5] Dai, J.X., Chung, M.S., Qu, R.M., Yuan, L., Liu, S.W. and Shin, D.S. (2012) The Visible Human Projects in Korea and China with Improved Images and Diverses Applications. Surgical and Radiologic Anatomy, 34, 527-534. https://doi.org/10.1007/s00276-012-0945-8

[6] Kim, C.H., Choi, S.H., Jeong, J.H., Lee, C. and Chung, M.S. (2008) HDRK-Man. A Whole-Body Voxel Model Based on High-Resolution Color Slice Images of a Korean Adult Male Cadaver. Physics in Medicine and Biology, 53, 4093-4106. https://doi.org/10.1088/0031-9155/53/15/006 
[7] Park, H.S., Chung, M.S., Shin, D.S., Jung, Y.W. and Park, J.S. (2013) Accessible and Informative Sectioned Images, Color-Coded Images, and Surface Models of the Ear. Anatomical Record, 296, 1180-1186. https://doi.org/10.1002/ar.22719

[8] Park, J.S., Chung, M.S., Hwang, S.B., Lee, Y.S., Har, D.H. and Park, H.S. (2005) Visible Korean Human: Improved Serially Sectioned Images of the Entire Body. IEEE Transactions on Medical Imaging, 24, 352-360. https://doi.org/10.1109/TMI.2004.842454

[9] Park, J.S., Chung, M.S., Hwang, S.B., Lee, Y.S., Har, D.H. and Park, H.S. (2005) Technical Report on Semiautomatic Segmentation by Using the Adobe Photoshop. Journal of Digital Imaging, 18, 333-343. https://doi.org/10.1007/s10278-005-6704-1

[10] Park, J.S., Chung, M.S., Hwang, S.B., Shin, B.S. and Park, H.S. (2006) Visible Korean Human: Its Techniques and Applications. Clinical Anatomy, 19, 216-224. https://doi.org/10.1002/ca.20275

[11] Park, J.S., Chung, M.S., Shin, D.S., Har, D.H., Cho, Z.H., Kim, Y.B., Han, J.Y. and Chi, J.G. (2009) Sectioned Images of the Cadaver Head Including the Brain and Correspondences with Ultrahigh Field 7.0 T MRIs. Proceedings of the IEEE, 97, 1988-1996. https://doi.org/10.1109/JPROC.2009.2025524

[12] Quackenbush, D., Ratiu, P. and Kerr, J. (1996) Segmentation of the Visible Human Data Sets. The Visible Human Project Conference Proceedings, France, 7-8 October 1996, 7-8.

[13] Schiemann, T., Freudenberg, J., Pflesser, B., Pommert, A., Priesmeyer, K., Riemer, M., Schubert, R., Tiede, U. and Höhne, K.H. (2000) Exploring the Visible Human Using the VOXEL-MAN Framework. Computerized Medical Imaging and Graphics, 24, 127-132. https://doi.org/10.1016/S0895-6111(00)00013-6 